\title{
Redundant regulation of meristem identity and plant architecture by
} FRUITFULL, APETALA1 and CAULIFLOWER

\author{
Cristina Ferrándiz¹, Qing Gu ${ }^{2, *}$, Robert Martienssen² and Martin F. Yanofsky ${ }^{1, \neq}$ \\ ${ }^{1}$ Department of Biology, University of California at San Diego, La Jolla, CA 92093-0116, USA \\ ${ }^{2}$ Cold Spring Harbor Laboratory, PO Box 100, Cold Spring Harbor, NY 11724, USA \\ *Present address: M409-WSL, Center for Legume Research and Division of Biology, University of Tennessee, Knoxville, TN 37996-0845, USA \\ ¥Author for correspondence (e-mail: marty@ucsd.edu) \\ Accepted 3 December 1999; published on WWW 26 January 2000
}

\section{SUMMARY}

The transition from vegetative to reproductive phases during Arabidopsis development is the result of a complex interaction of environmental and endogenous factors. One of the key regulators of this transition is $L E A F Y(L F Y)$, whose threshold levels of activity are proposed to mediate the initiation of flowers. The closely related APETALAI (AP1) and CAULIFLOWER (CAL) meristem identity genes are also important for flower initiation, in part because of their roles in upregulating $L F Y$ expression. We have found that mutations in the FRUITFULL (FUL) MADS-box gene, when combined with mutations in $A P 1$ and $C A L$, lead to a dramatic non-flowering phenotype in which plants continuously elaborate leafy shoots in place of flowers. We demonstrate that this phenotype is caused both by the lack of $L F Y$ upregulation and by the ectopic expression of the TERMINAL FLOWER1 (TFL1) gene. Our results suggest that the $F U L, A P 1$ and $C A L$ genes act redundantly to control inflorescence architecture by affecting the domains of $L F Y$ and $T F L 1$ expression as well as the relative levels of their activities.

Key words: Inflorescence meristem, Flowering time, Developmental phases, Flower development, MADS-box, $L E A F Y(L F Y)$,

TERMINAL FLOWER1 (TFL1), APETALA1 (AP1), CAULIFLOWER (CAL), FRUITFULL (FUL)

\section{INTRODUCTION}

Postembryonic development in Arabidopsis proceeds through a series of phases, each characterized by the identity of the lateral primordia produced by the shoot apical meristem (SAM) (Poethig, 1990). During the vegetative phase, the SAM produces closely spaced leaf primordia, each subtending a secondary shoot meristem, to form a rosette. During the reproductive, or inflorescence (I) phase, the SAM produces determinate floral meristems on its flanks. The last few vegetative leaves produced are referred to as cauline leaves and become separated along the inflorescence stem by longer internode distances. Thus, the production of leaves can be considered to occur within two distinct subphases, $\mathrm{V}_{1}$ (rosette) and $\mathrm{V}_{2}$ (cauline).

Genes that promote flowering in Arabidopsis were identified as mutations that extend the duration of the $\mathrm{V}$ phase, increasing the number of leaves formed before the development of flowers, but generally not affecting the fate of the lateral primordia produced during the I phase (reviewed by Piñeiro and Coupland, 1998). Another group of genes, including TERMINAL FLOWERl (TFL1), act by delaying phase change and preventing the normally indeterminate SAM from becoming a flower (Alvarez et al., 1992; Shannon and MeeksWagner, 1991). In addition, several meristem-identity genes are responsible for conferring floral characteristics to the lateral primordia produced by the SAM during the I phase. Mutations in floral meristem identity genes cause primordia that would develop into flowers to instead develop shoot characteristics. The best characterized of these genes are $L E A F Y$ ( $L F Y$ ), APETALA1 (AP1), APETALA2 (AP2) and CAULIFLOWER (CAL) (for review, see Yanofsky, 1995). Only lfy and apl mutants show dramatic flower-to-shoot phenotypes, especially in the most basal nodes. Furthermore, the nearly complete conversion of flowers into shoots observed in lfy apl double mutants reveals that they act redundantly to specify meristem fate (Bowman et al., 1993; Huala and Sussex, 1992; Irish and Sussex, 1990; Schultz and Haughn, 1991; Shannon and MeeksWagner, 1993; Weigel et al., 1992). Together, the LFY, AP1, $C A L$ and $A P 2$ genes appear to reinforce each other's activities leading to a sharp transition from vegetative to reproductive development.

The FRUITFULL (FUL) gene encodes a MADS-box protein that has previously been shown to be required for carpel and fruit development ( $\mathrm{Gu}$ et al., 1998; Mandel and Yanofsky, 1995a). However, in addition to its expression domain during carpel and fruit development, the FUL gene is upregulated in the SAM at around the transition to flowering, suggesting that it may also play a role during this transition (Mandel and Yanofsky, 1995a; Hempel et al., 1997). FUL is closely related to the meristem identity genes $A P I$ and $C A L$, suggesting the possibility of functionally redundant activities. 
In this work we have undertaken a molecular genetic approach to uncover the possible roles of $F U L$ in the transition to flowering as well as its interactions with different meristem identity genes. We have found that in addition to its role during carpel and fruit development, $F U L$ acts as a flowering-time and meristem-identity gene. These studies provide new insights into the functional redundancy of MADS-box genes during the transition to flowering and on the upregulation of the $L F Y$ meristem identity gene.

\section{MATERIALS AND METHODS}

\section{Plant material and growth conditions}

The ap1-1, ful-1, tfl-2 and lfy-26 alleles have been described previously (Bradley et al., 1997; Gu et al., 1998; Lee et al., 1997; Mandel et al., 1992). The cal-5 allele was generated in a $\gamma$-irradiation mutagenesis experiment and contains a single base-pair deletion $33 \mathrm{bp}$ downstream of the translation initiation codon that causes a frame shift and introduces a STOP codon 19 amino acids later (Savidge, 1996). 35S::LFY lines (DW151.2.5, in Landsberg erecta background; Weigel and Nilsson, 1995) and LFY::GUS (DW150.209, in Columbia; Blázquez et al., 1997) were kindly provided by Detlef Weigel. The 35S::AG line was obtained from Hong Ma (Mizukami and Ma, 1992). For all experiments, seeds were vernalized for $3-5$ days at $4^{\circ} \mathrm{C}$, then germinated and grown at $22-24^{\circ} \mathrm{C}$ under continuous light conditions.

\section{Characterization of the molecular lesions in the ful alleles}

For ful-2, ful-4, ful-5 and ful-6, genomic DNAs were amplified by PCR with the primers OAM25 (5'-GGTCATTTCAGGGTTGTCGGTT-3') and OAM14 (5'-AATCATTACCAAGATATGAA-3'), which hybridize respectively 59 ncl upstream of the initiation codon and 202 ncl downstream of the STOP codon of the FUL gene. The amplification products of two independent reactions were sequenced and compared with the wild-type sequence for each allele. For ful-5, since the sequencing of the FUL genomic DNA only showed a silent change in the coding region, we analyzed the sequence of the transcribed RNA by performing a reverse transcription of the ful-5 RNA using OAM14 as a primer, coupled with a PCR amplification using OAM25 and OAM14 as primers.

\section{GUS activity measurements}

For quantitative measurements of GUS activity in LFY::GUS ful-2 plants, the assay described by Blázquez et al. (1997) was used.

\section{In situ hybridizations}

For in situ experiments at day 12 in apl cal and ful apl cal plants, genotyping for the presence of the ful- 1 allele was necessary since double and triple mutants were indistinguishable (see below).

Tissue was fixed for 2 hours at room temperature in FAE solution (ethanol:acetic acid:formaldehyde:water, 50:5:3.5:41.5, v/v/v/v), dehydrated, embedded and sectioned to $8 \mu \mathrm{m}$. After dewaxing in histoclear and rehydration, sections were treated for 20 minutes in 0.2 $\mathrm{M} \mathrm{HCl}$, neutralized for 10 minutes in $2 \times \mathrm{SSC}$ and incubated for 30 minutes with $1 \mu \mathrm{g} / \mathrm{ml}$ Proteinase $\mathrm{K}$ at $37^{\circ} \mathrm{C}$. Proteinase action was blocked with 5 minutes incubation in $2 \mathrm{mg} / \mathrm{ml}$ Gly and 10 minutes postfixation in $4 \%$ formaldehyde. Tissue sections were washed in PBS, dehydrated through an ethanol series and dried under vacuum before applying the hybridization solution $(100 \mu \mathrm{g} / \mathrm{ml}$ tRNA; $6 \times \mathrm{SSC}$; $3 \%$ SDS; $50 \%$ formamide, containing approx. $100 \mathrm{ng} / \mu \mathrm{l}$ of antisense DIG-labeled RNA probe). Sections were hybridized overnight at $52^{\circ} \mathrm{C}$, washed twice for 90 minutes in $2 \times \mathrm{SSC} ; 50 \%$ formamide at $52^{\circ} \mathrm{C}$ and the antibody incubation and color detection was performed according to the manufacturer instructions (Boehringer). The probes were synthesized as previously described using plasmids pDW122
(LFY; Weigel et al., 1992), pCIT565 (AG; Drews et al., 1991), pD793 (AP3, Jack et al., 1992) and pSL66 (TFL1; Liljegren et al., 1999).

For double labeling experiments, a DIG-labeled TFL1 probe and a fluorescein-labeled $L F Y$ probe were both added to the hybridization solution. Washes, DIG-antibody incubation and color detection with NBT-BCIP as substrates were performed as described above to reveal TFL1 expression as a blue precipitate. Slides were treated in $2 \times$ SSC for 2 hours at $65^{\circ} \mathrm{C}$ to inactivate the alkaline phosphatase coupled to the DIG-antibody and then incubated with fluorescein-antibody. Color detection was performed according to the manufacturer instructions (Boehringer) using as a substrate Fast Red tablets to reveal $L F Y$ expression as a red signal.

\section{Scanning electron microscopy (SEM)}

Inflorescences were collected, fixed and observed as previously described (Gu et al., 1998).

\section{Generation and identification of multiple mutants}

In all combinations the ful- 1 allele was used, except for the generation of LFY::GUS ful-2 lines. ful-1 carries a Ds:GUS element that allows the identification of the mutant allele by assaying GUS activity in cauline leaves. ful- 1 was crossed as female to homozygous mutants ap1-1, apl-1 cal-5 and tfl1-2 and double/triple mutants were identified in $F_{2}$ populations as new/additive phenotypes segregating in a 1:16 ratio (1:64 for ful apl cal).

Since $L F Y$ and $F U L$ are closely linked (approximately $1 \mathrm{cM}$ ), ful1 pollen was crossed onto lfy-26 homozygous plants and GUS detection was performed on $\mathrm{F}_{2}$ plants with lfy phenotype until one positive was found, and pollinated with ful- 1 pollen. In the $F_{1}$ from this cross, plants with ful phenotype were selected as ful-1 lfy-26/+ and selfed, and $\mathrm{F}_{2}$ lfy plants were assumed to be homozygous for both mutations.

lfy-26 ap 1-1 cal-5 ful-1 were generated by crossing ap1-1 cal-5 onto ful-1 lfy-26. Plants with apl cal phenotypes in the $\mathrm{F}_{2}$ generation were tested for GUS activity, and the positives were selfed and assumed to have an apl cal (ful lfy)/++ genotype. The $\mathrm{F}_{3}$ populations resulting from these selfed individuals segregated plants with apl cal and apl lfy-like phenotypes in a 3:1 ratio, and the latter ones were assumed to be the quadruple mutant combination, since given the close linkage between $L F Y$ and $F U L, 99 \%$ of the $\mathrm{F}_{3}$ lfy plants would be $f u l$ homozygous.

For the generation of $t f l-2$ apl-1 cal-5 ful-1 quadruple mutants, tfl1-2 ful-1 plants were used as females for ap 1-1 cal-5 pollen. ful phenotypes were selected and selfed in the $F_{1}$ generation and in the $\mathrm{F}_{2}$, since no new phenotypes were observed, the presence of the cal5 allele was analyzed in plants with apl ful t fl phenotype as described below.

For 35S::LFY apl-1 cal-5 ful-1, 35S::LFY plants were used as pollen donors to fertilize ap 1-1 cal-5 ful-1/+ emasculated flowers. 35S::LFY phenotypes with GUS activity were selected and selfed in the $\mathrm{F}_{1}$ generation, and in the $\mathrm{F}_{2}$, plants with ful fruit phenotypes were genotyped for the presence of 35S::LFY, ap1-1 and cal-5 and further reconfirmed by the segregation of the selfed $\mathrm{F}_{3}$ progenies. For 35S::AG ap 1-1 cal-5 ful-1, the same strategy was adopted, except that in the $F_{2}$ population, a new phenotype was identified as the quadruple combination.

\section{Genotyping}

To genotype plants for the presence of the ap1-1 allele, we used dCAPS markers (Neff et al., 1998). The ap 1-1 mutation introduces a change from $\mathrm{G}$ to $\mathrm{A}$ at the acceptor site before exon 4 (Mandel et al., 1992). For genotyping, genomic DNA was amplified by PCR using the primers 5'-GCAAGTCTTCCCCAAGATAAGGC-3' and 5'GACAGCTTATTGCACCTGAG-3'. The restriction enzyme StuI cleaves only wild-type DNA yielding a 290 and a 22 bp fragment.

For cal-5 genotyping, a FokI RFLP was used. Genomic DNA was amplified by PCR with primers 5'-ATGGGAAGGGG-TAGGGTTG- 
$3^{\prime}$ and $5^{\prime}$-ATTCAGAG-GAGTACTCGAAC-3'. Digestion with FokI generates two fragments of 42 and $140 \mathrm{bp}$ in cal-5 DNA, while wildtype DNA is not digested.

ful-1 plants were genotyped by PCR on genomic DNA with the primer AGL8PG: 5'-TGTATTCACGTCACATACCG-3', located in the promoter region of the $F U L$ gene, and primers AGL8MG: 5'-CTCATGAGC-TTTCTTGAGC-3' and GUS3: 5'-CTTGTAACGCGCTTTCCC- $3^{\prime}$, located respectively in the coding regions of the FUL gene and the GUS gene of the Ds-element inserted in the ful- 1 allele. ful- 1 homozygotes were identified by the detection of an amplification product with primers AGL8PG/GUS3, and no product with the pair AGL8PG/AGL8MG.

The presence of the 35S:LFY transgene was assessed by PCR on genomic DNA with the primers 5'-ACCCAAGCTTCTTCGCAAGACCCTTCCTCT-3', located in the $35 \mathrm{~S}$ promoter region, and $5^{\prime}$ AACTAGAAACGCAAGTCG-3', located in the $L F Y$ coding region, which only amplified the transgene and no endogenous sequences.

\section{RESULTS}

\section{The fruitfull phenotypes}

The ful-1 mutation affects several aspects of plant development. The most dramatic effect is observed within the carpel where, from stage 12 (Smyth et al., 1990), the valve cells fail to elongate and differentiate, and the replum cells grow with an altered morphology. As a result, silique development is severely affected, leading to short fruits with small crowded seeds (Fig. 1A,J,N; Gu et al., 1998). In addition to the fruit phenotype, ful- 1 cauline leaves are also affected in shape, cell organization and vascular differentiation ( $\mathrm{Gu}$ et al., 1998).

We analyzed five new ful alleles (see Materials and Methods) and examined their phenotypes. ful-4, ful-5 and ful-6 were isolated after EMS mutagenesis in the Landsberg erecta (Ler) background. The strong ful-5 allele, which has a phenotype very similar to ful- 1 , has a single base pair change from $\mathrm{C}$ to $\mathrm{T}$ in position 156 of the first exon. This mutation results in the generation of an alternate donor splicing site at position 154 that causes a 31 base deletion in the messenger RNA, a frame shift and a truncated protein. ful-4 and ful-6 contain single base pair mutations from $\mathrm{G}$ to $\mathrm{A}$ in the $5^{\text {th }}$ and $6^{\text {th }}$ exon-intron boundaries respectively. ful-4 has an intermediate phenotype, with a similar arrested development of the valves, but a less pronounced defect in replum morphology (Fig. 1D,L,P). ful-6 is a

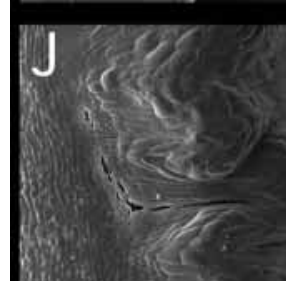

ful-1 weak allele, which causes the valve cells differentiate and expand to almost the same extent as the wild type, and the replum cells undergo mild enlargement, allowing the formation of a functional dehiscence zone in the valve-replum boundaries (Fig. 1E,M,Q).

As a result of these two alleles a striking elongation of the style was observed in genetic backgrounds different from Ler. ful-2 is an EMS mutagenized line in the Columbia $(\mathrm{Col})$
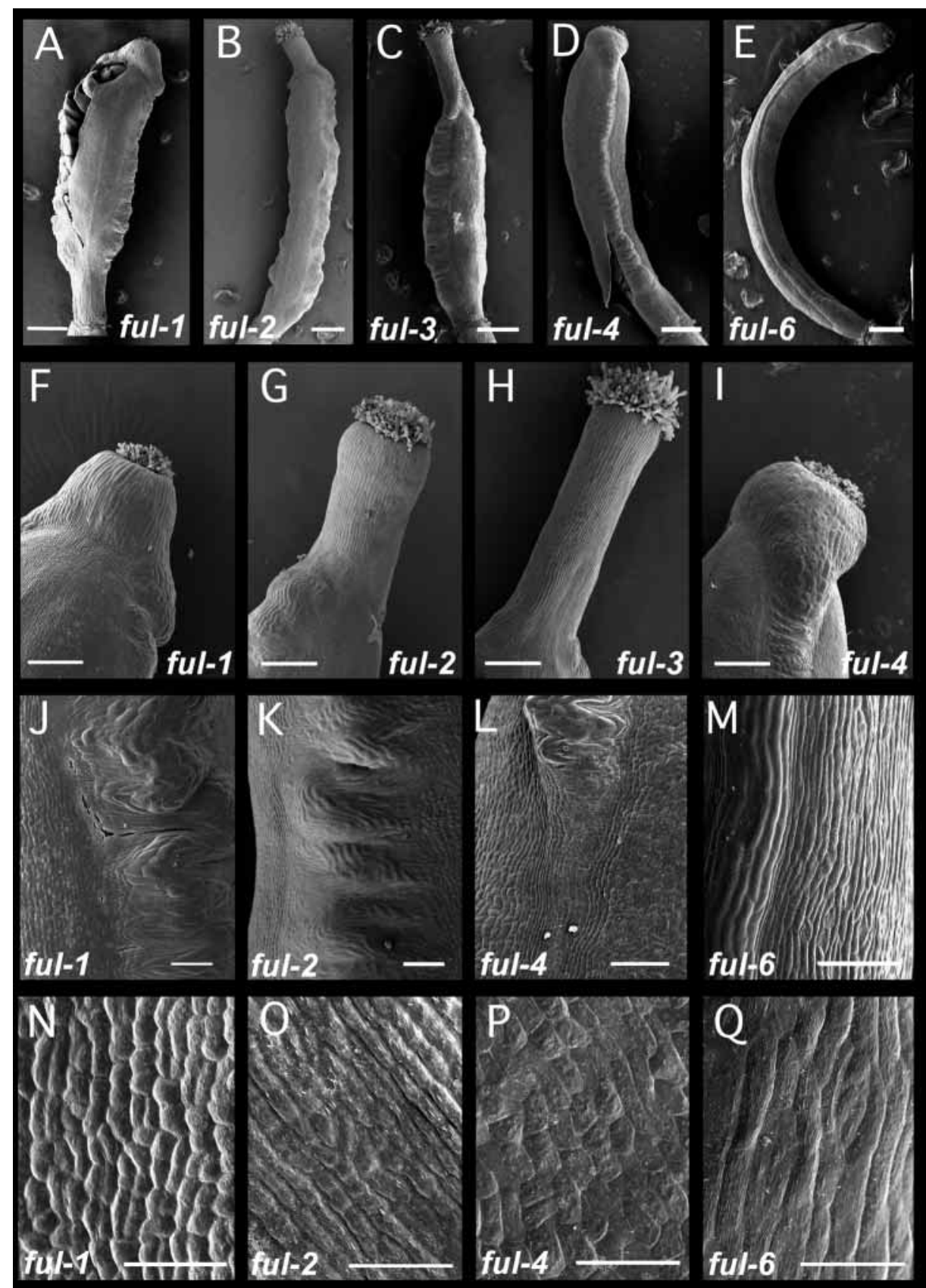

fult

tul-4

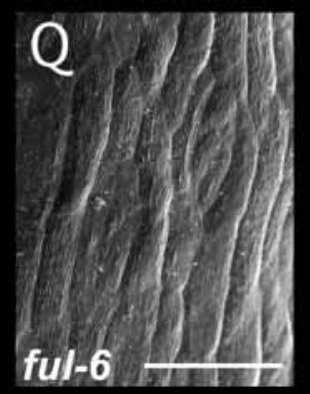

Fig. 1. Scanning electron micrographs of fruitfull mutants showing fruits 10 days after pollination (late stage 17; according to Smyth et al., 1990). (A-E) ful allelic series. (F-I) Style phenotypes from four different $f u l$ alleles. (F and I) ful- 1 and ful-4 respectively, both in the Landsberg erecta background. (G) A ful-2 style, in Columbia background and (H) a ful-3 style, in Nossen background. Close-up of (J-M) the valve-replum boundary and (N-Q) the valve epidermal cells in four different $f u l$ mutants as indicated. Scale bars, $500 \mu \mathrm{m}$ (A-E), $200 \mu \mathrm{m}$ (F-I), $100 \mu \mathrm{m}(\mathrm{J}-\mathrm{M})$ and $50 \mu \mathrm{m}(\mathrm{N}-\mathrm{Q})$. 
Table 1. Time to flowering under continuous light conditions

\begin{tabular}{lccc}
\hline Genotype & $\begin{array}{c}\text { No. } \\
\text { rosette leaves }\end{array}$ & $\begin{array}{c}\text { No. } \\
\text { cauline leaves }\end{array}$ & Total leaves \\
\hline Landsberg erecta & $7.9 \pm 0.6$ & $2.9 \pm 0.4$ & $10.8 \pm 0.6$ \\
ful-1* & $9.7 \pm 0.4$ & $4.2 \pm 0.3$ & $13.9 \pm 0.4$ \\
Columbia & $11.3 \pm 1.0$ & $3.3 \pm 0.3$ & $14.3 \pm 1.1$ \\
ful-2 & $12.9 \pm 1.1$ & $5.0 \pm 0.5$ & $17.9 \pm 1.2$ \\
lfy-26* & $8.7 \pm 0.7$ & $7.1 \pm 1.1$ & $15.8 \pm 1.2$ \\
ful-1 lfy-26* & $9.9 \pm 0.5$ & $10.2 \pm 1.1 \ddagger$ & $20.3 \pm 1.1$ \\
LFY/lfy-26* & $9.0 \pm 0.5$ & $3.9 \pm 0.3$ & $12.9 \pm 0.5$ \\
ful-1 LFY/lfy-26* & $9.5 \pm 0.6$ & $5.9 \pm 0.5 \ddagger$ & $15.4 \pm 0.5$
\end{tabular}

Flowering time is expressed as the number of leaves produced by the main shoot.

LFY/lfy-26 and ful-1 LFY/lfy-26 plants were identified by genotyping of the progenies of segregating populations with a CAPS marker (see Materials and Methods).

Values are mean \pm standard error. $n \geqslant 15$ in all cases.

*All in Landsberg erecta background.

$\ddagger$ Refers to the number of leaves subtending shoots.

background, and contains a nonsense mutation in exon 3 (W91 to STOP). ful-3 is a Nossen line with a Spm transposon element inserted into the last intron. The ful-2 and ful-3 styles of late stage 17 fruits are 2-3 times longer than the Col wild type (Fig. 1F-H). ful-2 siliques have a less severe phenotype in the valves, whose cells elongate to a small extent allowing a modest expansion of the fruit, although, as in ful-1, no differentiation of stomata is observed. ful-3 is a strong allele producing a similar phenotype to ful- 1 in valves and replum. Even though a gradation in the silique phenotypes was observed in the allelic series, the cauline leaves in all mutants were affected similarly, suggesting that the FUL function in leaf morphology requires its complete activity (results not shown).

\section{Mutations in FRUITFULL affect flowering time}

Since $F U L$ is strongly upregulated in the shoot apical meristem shortly after the transition to flowering, and in response to photoinductive conditions, a possible role for FUL in promoting the initiation of flowers has been proposed (Hempel et al., 1997; Mandel and Yanofsky, 1995a). To investigate this possibility, we quantified flowering time in ful mutants grown under continuous light. ful plants showed a small, significant delay in the time to flower when measured both in number of leaves (Table 1) and days to bolt (results not shown). The increase in leaf number was observed for both rosette and cauline leaves, and no significant effect of the erecta mutation was observed, since both ful-1 (Ler) and ful-2 (Col) mutants flowered later than the corresponding wild-type ecotypes. These results suggest that $F U L$ acts to promote the flowering pathway, although, given the slight effect in flowering time, it may be involved in a highly redundant network of signaling.

\section{$F U L$ and $L F Y$ act in parallel pathways}

One candidate for a gene that interacts with $F U L$ in this flowerpromoting pathway is $L F Y$, as $L F Y$ levels have been shown to be important for the transition to flowering (Blázquez et al., 1997). lfy mutants have an extended $V_{2}$ phase and are defective in floral meristem identity specification (Huala and Sussex, 1992; Schultz and Haughn, 1991; Weigel et al., 1992). In response to photoinductive treatments, $L F Y$ and $F U L$ are
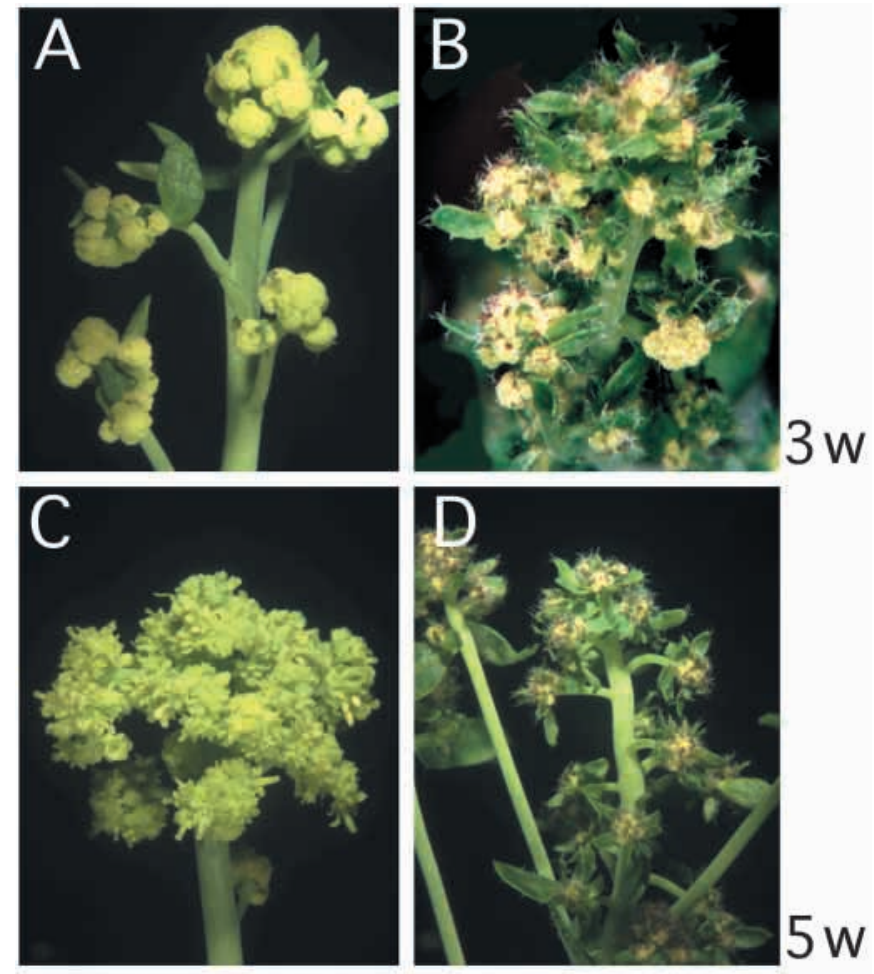

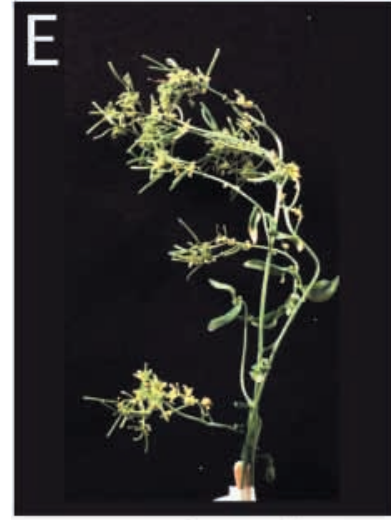

ap $1 \mathrm{cal}$

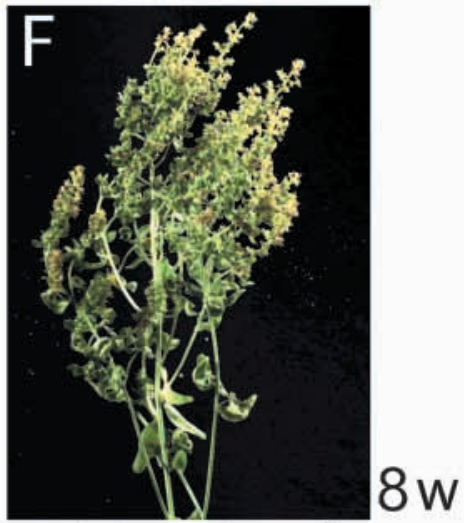

ful ap 1 cal
Fig. 2. Mutant phenotypes of ful apl cal and apl cal plants. (A-B) Apical inflorescences of 3-week-old apl cal (A) and ful apl cal (B) plants. (C-D) Apical inflorescences of 5-week-old apl cal (C) and ful apl cal (D) plants. Mature floral organs are visible in apl cal, but absent in ful apl cal inflorescences. (E) An apl cal plant, 8 weeks after germination. Mature siliques have already developed. (F) A ful apl cal plant, 8 weeks after germination. The inflorescence structures have branched out producing numerous leafy shoots, but no flower structures are evident.

upregulated at the shoot apex in an overlapping pattern (Blázquez et al., 1997; Hempel et al., 1997).

To investigate whether the late flowering phenotype in ful mutants might be caused by a delay in $L F Y$ upregulation or a reduction in its levels, we introduced a LFY::GUS transgene into ful-2 plants (see Methods). We did not observe any effect of the ful mutation on the upregulation of $L F Y$ (not shown), suggesting that either $L F Y$ is not directly regulated by $F U L$, or that other genes can substitute for $F U L$ to upregulate $L F Y$. In addition, we generated ful-1 lfy-26 double mutants, which 

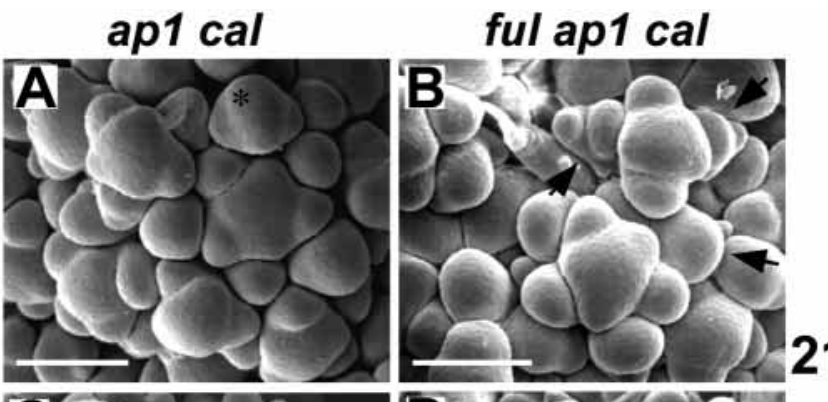

Fig. 3. Scanning electron micrographs of apl cal (A,C) and ful apl cal (B,D) apical meristems. In 21-day-old apl cal inflorescences (A), floral meristems (*) up to stage 3 of development (according to Smyth et al., 1990) are visible. ful apl cal apices of the same age (B) initiate a higher number of cauline leaves with axillary meristems (arrows) and no floral meristems are visible. 35-day-old apl cal inflorescences (C) are composed of floral meristems at several developmental stages $(*)$, some with characteristics of inflorescence meristems, such a spiral phyllotaxy. 35-day-old ful apl cal apices (D) show a proliferation of mainly leaf primordia with axillary meristems (arrows), with no recognizable phyllotactic arrangement.
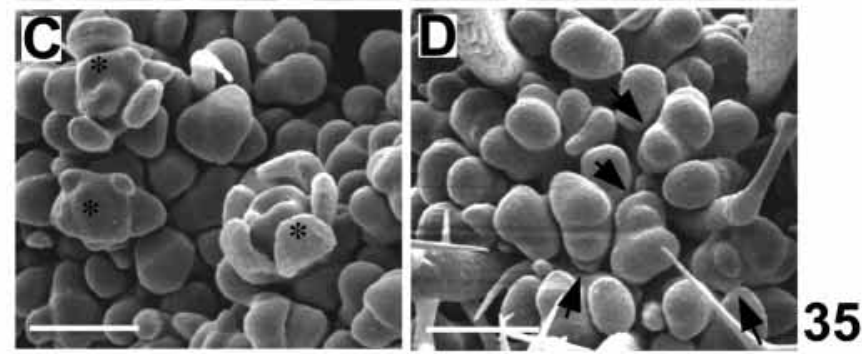

Scale bars, $100 \mu \mathrm{m}$.

plants, with a more dramatic increase in the duration of the $\mathrm{V}_{2}$ phase as scored by the number of cauline leaves produced (Table1), suggesting that $L F Y$ and $F U L$ act in parallel pathways or at least influence the same process independently. ful-l lfy26 plants also showed an additive phenotype in the floral identity defects, with the same kind of flower-to-shoot transformations as in $l f y-26$, and the ful- 1 phenotype in the carpel-like organs that eventually formed (not shown).

flowered later than the corresponding lfy-26 and ful- 1 single mutants (Table 1). Moreover, a similar quantitative effect was observed in ful-1 plants heterozygous for lfy-26, which flowered significantly later than either ful-1 mutants or LFY/lfy

\section{Flowering is eliminated in ful ap1 cal triple mutants}

The APl gene is required for both flower meristem and flower organ identity. (Irish and Sussex, 1990; Bowman et al., 1993).
Fig. 4. Expression of $T F L 1$ and $L F Y$ in wildtype, apl cal and ful apl cal plants. Sections of wild-type apices at day 14 (A), apl cal plants at days 14 (B), 21 (D) and 35 (F) and ful apl cal plants at days 14 (C), 21 (E) and 35 (G) probed simultaneously with $L F Y$ (red label) and TFLl (blue label) antisense RNA. In day-14 wild-type plants (A) $L F Y$ is strongly expressed in flower meristems arising laterally from the inflorescence apex. TFL1 is expressed below the inflorescence meristem in a region not overlapping with $L F Y$. In day-14 apl cal plants (B), $L F Y$ is weakly expressed and $T F L 1$ is ectopically detected below the lateral meristems initiated. $L F Y$ and $T F L 1$ domains of expression overlap in some regions, as described by Ratcliffe et al. (1999). In day-14 ful apl cal meristems (C), $L F Y$ and $T F L 1$ expression is similar to apl cal at the same time point. In day-21 apl cal inflorescences (D,E), $L F Y$ is detected at high levels in some meristems, presumably those already committed to a floral fate. TFL1 is not found in highly $L F Y$-expressing meristems, and is strongly detected in those putatively behaving as inflorescence meristems. Some regions still show the early pattern of $L F Y$ and TFL1 coexpression. Day21 ful ap 1 cal plants still show very low levels of $L F Y$ expression, and TFL1 can be detected below most of the meristems initiated, in a similar pattern to earlier time points. At 35 days, apl cal inflorescences show high $L F Y$ expression in floral meristems and $T F L$ is barely detectable. In 35-day-old ful apl cal plants (G), $L F Y$ can be detected at similarly low levels, whereas TFL1 expression is reduced and restricted to a few meristems.
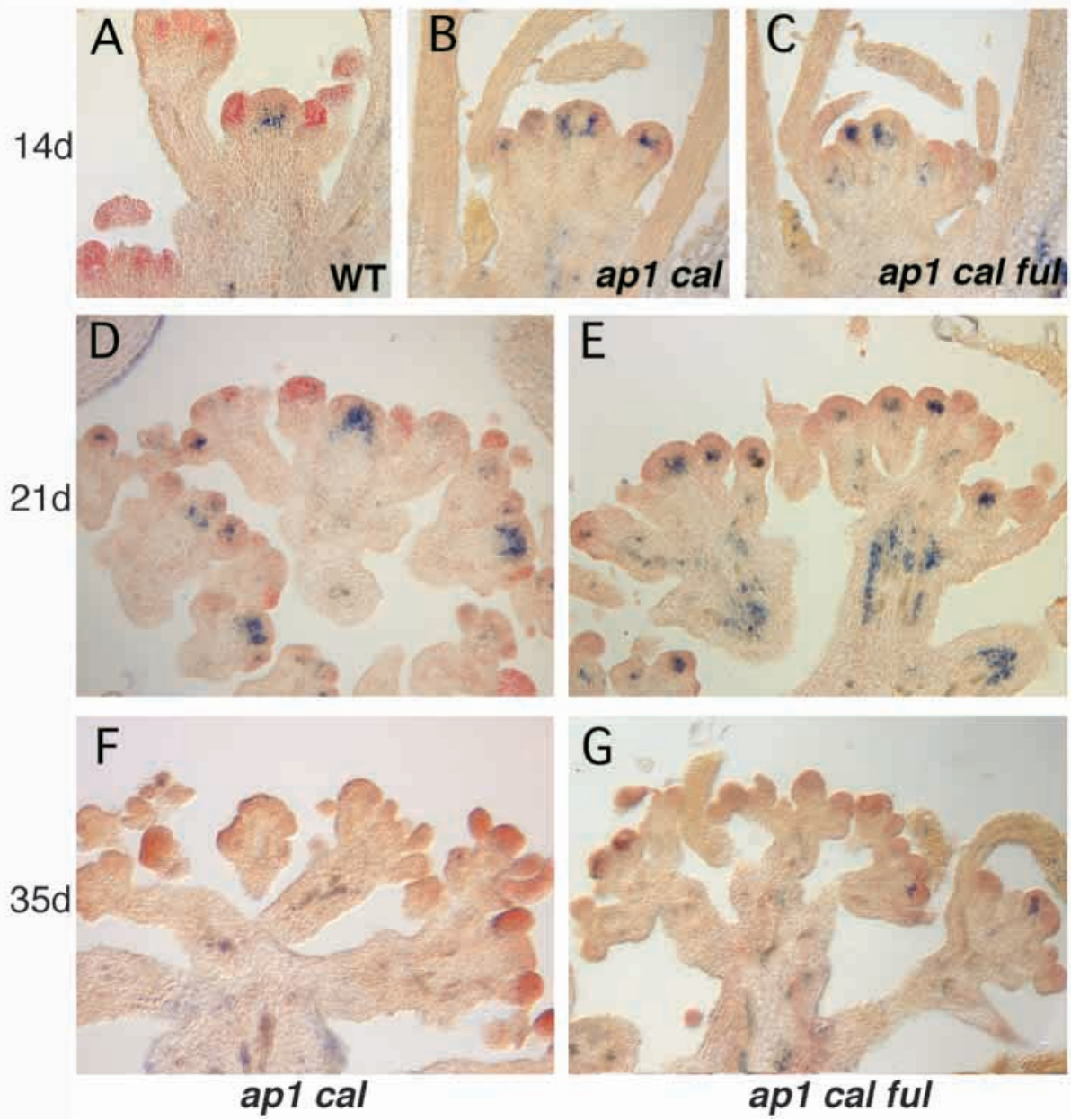
Mutations in the closely related and partially redundant $C A L$ gene enhance the apl mutant phenotype, such that apl cal double mutants proliferate inflorescence meristems in positions that would normally be occupied by flowers, resulting in a 'cauliflower' appearance. Eventually, however, flower meristems are specified and apl-like flowers are produced (Bowman et al., 1993; Kempin et al., 1995; Fig. 2A,C,E). The APl gene also plays a role in negatively regulating $F U L$ in emerging flower primordia, as FUL is ectopically expressed in apl and apl cal mutant flower meristems (Mandel and Yanofsky, 1995a; M. A. Mandel, C. F. and M. F. Y, unpublished observations).

The close sequence similarity of FUL, API and CAL (Mandel and Yanofsky, 1995a), together with the fact that $F U L$ is expressed throughout the proliferating meristems of apl cal double mutants, suggested the possibility that the ability of apl cal mutants to eventually form flowers could be the result of ectopic $F U L$ activity. In order to test this hypothesis, we introduced the ful-1 mutation into ap $1-1$ cal5 mutant plants. We found that mutations in ful dramatically enhanced the apl cal double mutant phenotype such that the triple mutant plants proliferated leafy shoots and failed to flower. After bolting, the ful apl cal shoot apical meristem gave rise to cauline leaves with associated axillary meristems which repeated this pattern to form leafy shoots with small axillary 'cauliflowers' along the main inflorescence, which as a consequence, branched out extensively to adopt a bushy appearance (Fig. 2B,D,F).

A closer inspection by SEM of apl cal and ful apl cal inflorescences revealed differences in the behavior of the proliferating meristems. Early in their development, apl cal double mutants displayed the conversion of flowers into inflorescence meristems. In 21-day-old plants, fifth order inflorescence meristems arranged in a spiral phyllotaxy were apparent and some of the older meristems had visibly acquired a flower identity (Fig. 3A). After 35 days of growth, flowers with developing stamens and carpels were visible in the lower branches, and in the more apical positions new flowers were differentiating (Figs 2C, 3C). After 2 months of growth, the meristems had stopped proliferating and fully developed siliques were observed (Fig. 2E).

By contrast, the ful apl cal meristems at 21 days typically produced cauline leaf primordia with associated axillary meristems as well as two or three additional meristems not subtended by leaves, yielding a phyllotaxy intermediate between spiral and cruciform (Fig. 3B). At these and all later time points, no floral structures were evident, and the proliferating meristems gave rise to new meristems and cauline leaves at a lower rate, losing all phyllotactic arrangement (Fig. 3D). The ful apl cal plants kept under our standard growth room conditions failed to produce any kind of flower structures even six months after germination, and remained in this vegetative state until they died. Interestingly, we did observe on one occasion, when accidental overheating of the growth room occurred, that some of these plants formed a few floral organs after several months of vegetative development. These results suggest that there may be a threshold required to induce flowering, and that although the triple mutant is normally just below this threshold, conditions such as heat stress can allow the requirement for $F U L / A P 1 / C A L$ to be overcome.

In contrast to the enhancement of the apl cal double mutant phenotype, we did not observe enhancement of the apl mutant phenotype when ful-1 apl-1 double mutants were generated. The phenotype of the double mutants was found to be strictly additive for both flowering time and flower and fruit development (results not shown). Likewise, ful-1 cal-5 double mutants were indistinguishable from the ful- 1 single mutants, consistent with previous observations showing that mutations in $C A L$ do not confer a mutant phenotype in the presence of a functional copy of $A P 1$. These results indicated that both AP1 and $C A L$ are able to compensate for the loss of $F U L$ function in specifying floral meristem identity.

\section{Overexpression of $L F Y$ rescues the non-flowering phenotype of ful ap1 cal plants}

The non-flowering phenotype of ful apl cal mutants is likely the result of reduced activity of the $L F Y$ meristem identity gene product, and there are two distinct explanations for how $F U L / A P 1 / C A L$ can contribute to LFY activity. One scenario is that $L F Y$ RNA expression in the ful apl cal triple mutant may not reach the threshold required for flower specification. An alternative scenario is that the activity of the LFY protein may require one of the AP1, CAL or FUL functions, for example as a cofactor, even though $L F Y$ RNA levels in the triple mutant may be sufficient to promote flowering.

To distinguish between these possibilities, we compared the expression of $L F Y$ in apl cal double mutants to that in ful apl cal triple mutants grown under continuous light. In both apl cal and ful apl cal plants 14 days after sowing (d14), LFY RNA levels were much lower than in the corresponding wild-type controls (Fig. 4A-C). In d21 apl cal inflorescences, floral meristems and flowers up to stage 5 of development could be easily distinguished, whereas in ful apl cal mutants, no floral characteristics appeared (Fig. 3A,B). LFY expression was detected at high levels in all floral meristems of apl cal plants. In contrast, $L F Y$ RNA levels were significantly reduced in $f u l$ apl cal triple mutants, although the accumulation of $L F Y$ RNA was still readily detected (Figs 4E, 6A,B). These results suggests that $A P 1, C A L$ and $F U L$ play a redundant role in boosting $L F Y$ RNA levels but that other factors are capable of inducing $L F Y$ expression initially.

The reduced levels of $L F Y$ RNA accumulation in the triple mutant provide molecular evidence that suggests that the failure to upregulate $L F Y$ may be the cause of the nonflowering phenotype. To demonstrate that this is indeed the case, we introduced a constitutively expressed $L F Y$ transgene (35S::LFY) into ful apl cal triple mutants. In contrast to the triple mutant, 35S::LFY ful apl cal plants were able to produce many ful apl-like flowers, indicating that high levels of $L F Y$ expression could overcome the lack of $A P 1 / C A L / F U L$ functions (Fig. 5A). Taken together, these data demonstrate that the inability of ful apl cal plants to flower is due to a reduction of $L F Y$ expression.

It has been previously described that apl mutations can largely suppress the early flowering phenotype conferred by the 35S::LFY transgene (Mandel and Yanofsky, 1995b; Weigel and Nilsson, 1995). The phenotypes of the 35S::LFY, 35S::LFY ap1, 35S::LFY apl cal and 35S::LFY ful apl cal showed a gradation in flowering time (Table 2) and number of flowers (not shown) produced by the shoot apical meristem before terminating. These results suggest that the early flowering and shoot-to-flower conversion caused by the 
Table 2. Effect of the ap1/cal/ful mutations on the 35S::LFY phenotype

\begin{tabular}{lccc}
\hline Genotype & $\begin{array}{c}\text { No. } \\
\text { rossette leaves }\end{array}$ & $\begin{array}{c}\text { No. } \\
\text { cauline leaves }\end{array}$ & Total leaves \\
\hline Landsberg erecta & $8.1 \pm 0.5$ & $2.3 \pm 0.6$ & $10.4 \pm 0.6$ \\
35S::LFY & $6.1 \pm 0.5$ & - & $6.1 \pm 0.5$ \\
35S::LFY ap1 & $6.8 \pm 0.5$ & $2.3 \pm 0.5$ & $9.1 \pm 0.5$ \\
35S::LFY ap1 cal & $8.9 \pm 0.6$ & $4.2 \pm 0.5$ & $13.1 \pm 0.6$ \\
35S::LFY ful ap1 cal & $9.3 \pm 0.6$ & $10.3 \pm 1.1$ & $19.3 \pm 1.1$
\end{tabular}

The number of leaves produced by the main shoot is used as a measure of flowering time.

Values are mean \pm standard error. $n \geqslant 15$ in all cases.

All in Landsberg erecta background.

35S::LFY transgene is mostly due to the subsequent activities of FUL/AP1/CAL.

Because low levels of $L F Y$ RNA accumulate in ful apl cal triple mutants, it was unclear if $L F Y$ was still active in the triple mutant. We therefore introduced the strong lfy-26 allele into the triple mutant and found the quadruple mutants to be very similar to lfy apl doubles or lfy apl cal triples (Bowman et al., 1993). The plants showed enhanced vegetative characteristics, developing secondary shoots in place of flowers, subtended by the typical ful-l cauline leaves ( $\mathrm{Gu}$ et al., 1998). The proliferation of 'leafy cauliflowers' that occurs in ful apl cal triple mutant does not occur in lfy ful apl cal quadruple mutants. Instead, the quadruple mutant develops vegetative shoots, indicating that some $L F Y$ activity is needed to cause a reiterative pattern of meristem proliferation. In addition, the similar phenotypes of lfy apl and lfy ful apl cal indicate that in an apl lfy background, FUL and $C A L$ are not able to specify floral meristem identity.

\section{Mutations in TFL1 suppress the nonflowering phenotype of ful ap1 cal plants}

A number of studies have demonstrated an antagonistic interaction between TFL1 and the floral meristem identity genes (Mandel and Yanofsky, 1995b; Savidge, 1996; Weigel and Nilsson, 1995; Weigel et al., 1992; Lijegren et al., 1999; Ratcliffe et al., 1999). To further explore these interactions, we compared the expression patterns of $T F L$ and $L F Y$ in the meristems of apl cal mutants to that in the ful apl cal triple mutants. $L F Y$ is strongly expressed in wild-type flower meristems 14 days after sowing (d14), but is only expressed at low levels in equivalently staged apl cal double mutants. One week later ( $\mathrm{d} 21)$, when flower meristems become distinct in apl cal mutants, $L F Y$ RNA is detected at higher levels and its expression increases at later time points when flowers are already apparent (d35) (Figs $4 \mathrm{~B}, \mathrm{D}, \mathrm{F}, 6 \mathrm{~A})$ ). In contrast, $L F Y$ is only detected at low levels in ful apl cal mutants, even after two months of growth (Figs 4C,E,G, 6B).

TFL1 is normally expressed in the center of the inflorescence apex and is not detected in the lateral meristems committed to a floral fate (Fig. 4A). TFL1 expression was found at comparable levels and pattern in apl cal and ful apl cal plants at d14, below the shoot apex and, ectopically, in the laterally arising meristems (Fig. 4B,C). Similar domains of TFL1 expression were found in apl cal and ful apl cal meristems at $\mathrm{d} 21$; in both cases, TFL1 was expressed more strongly in basal positions and at lower levels in the more apical meristems At d35, TFL1 was almost undetectable in apl cal inflorescences, where $L F Y$ expression had reached its highest level, whereas some small domains of $T F L$ expression and lower levels of $L F Y$ could still be seen in ful apl cal (Fig. 4F,G). Thus, the mutually exclusive patterns of $L F Y$ and TFLI expression became overlapping both in apl cal and ful apl cal meristems, even though the ratio of $L F Y$ to $T F L 1$ RNA expression was clearly higher in apl cal than in ful apl cal meristems from d21 (Fig. 4D,E).

Since TFL1 RNA accumulates in the ful apl cal triple mutant, we examined the activity of TFL1 in this context by introducing the tfll mutation into the triple mutant. ful apl cal tfl1 plants were only distinguishable from apl tfl1 plants by the ful fruit phenotype, indicating that the lack of TFL1 completely abolished the proliferation of meristems observed in ful apl cal plants (Fig. 5B). We also observed that, as in wild type, in the ful apl cal background, the tfll mutation had a semidominant phenotype. ful apl cal TFL1/tfll plants were initially indistinguishable from the ful apl cal triples, but, after 2 months of growth, they all were able to form some flowers and set seeds. The quantitative effect of TFL1 on the ful apl cal phenotype might reflect a threshold ratio of LFY:TFL1 activities that has to be reached to induce flowering.

\section{ful ap1 cal meristems are not competent to respond to $A G$ floral inductive activity}

We have shown that $L F Y$ RNA levels are significantly reduced in ful apl cal triple mutants, and previous studies have demonstrated that $L F Y$ plays a key role in the upregulation of the $A P 3$ and $A G$ organ identity genes. We therefore compared the expression of $A P 3$ and $A G$ in the ful apl cal triple mutant to that in the apl cal double mutant to determine if the expression of these organ identity genes requires $A P 1 / C A L / F U L$. Whereas the accumulation of $A P 3$ and $A G$ RNAs was readily detected in 21-day-old apl cal meristems, these RNAs were not detected in ful apl cal plants of the same age (Fig. 6C-F). Even after more than 2 months of growth (not shown), no expression of $A P 3$ and $A G$ could be detected in the triple mutant. These results demonstrate that $F U L, A P 1$ and $C A L$ have overlapping functions in the upregulation of $A P 3$ and $A G$, most likely mediated at least in part through the upregulation of $L F Y$.

These results raise the question of whether the inability to produce floral structures in ful apl cal mutants is due simply to the reduced $L F Y$ expression, or is due in part to the loss of organ-identity gene activation. Related to this is the observation that $A G$ promotes floral identity even in the absence of $L F Y$ and $A P l$ functions and is necessary to maintain floral identity under non-inductive conditions (Mizukami and Ma, 1997). To test whether the lack of the $A G$ floral promoting activity was the direct cause of the non-flowering phenotype, we introduced a 35S::AG transgene in ful apl cal plants. Constitutive expression of $A G$ did not induce the ful apl cal plants to flower, indicating that the loss of flowering in the triple mutant is not simply due to a failure to upregulate $A G$ (Fig. 5C,D) and that $A G$ requires at least one of the FUL/AP1/CAL activities to promote a floral fate.

\section{DISCUSSION}

\section{FUL controls several aspects of plant development}

We have shown that FRUITFULL is involved in several distinct processes during Arabidopsis development, as was suggested 

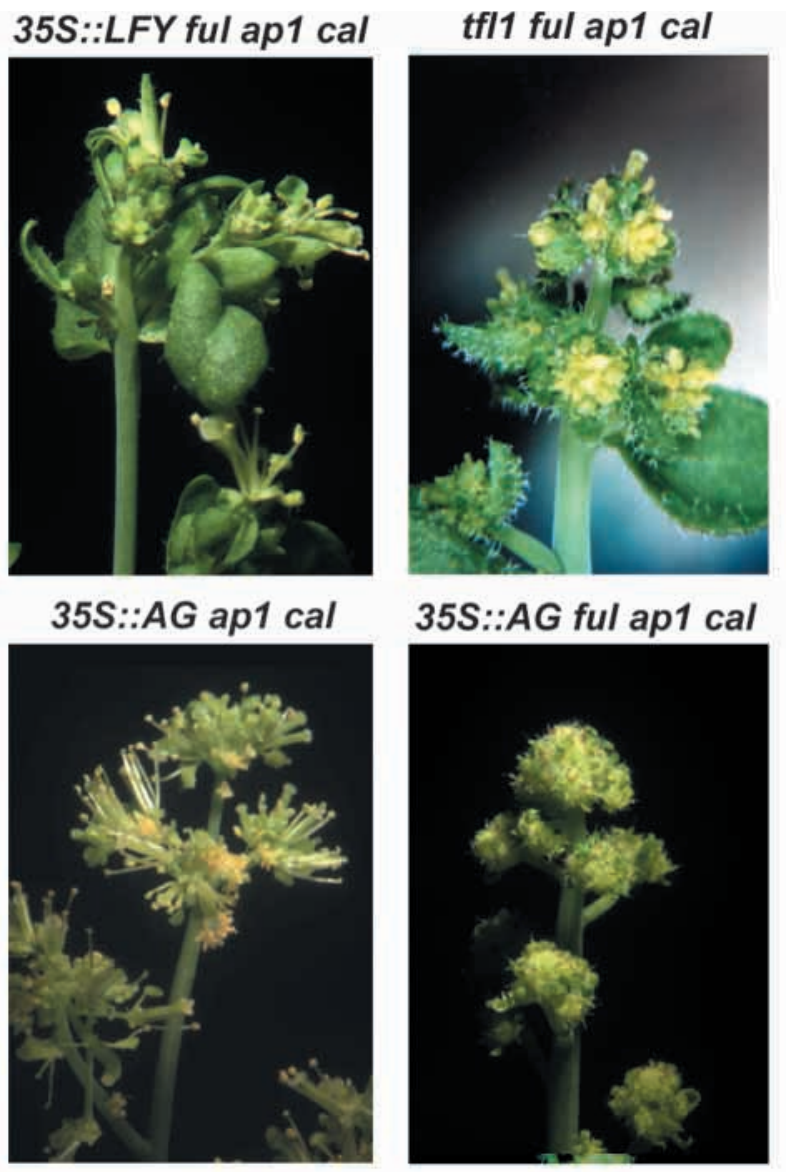

Fig. 5. Phenotype of the $35 S:: L F Y$ ful apl cal, tfl ful apl cal, $35 S:: A G$ apl cal and 35S::AG ful apl cal plants. All plants were grown for 4 weeks. (A) $35 S:: L F Y$ ful apl cal inflorescences produced flowers composed of bract-like sepals, stamens and ful-like carpels, subtended by typical ful cauline leaves. (B) The quadruple tfll ful ap $1 \mathrm{cal}$ mutants produced flowers similarly to tfl ap 1 plants. The 35S::AG transgene accelerates flowering in apl cal inflorescences (C), which form apl-like flowers without producing 'cauliflower' structures first (as described in Mizukami and Ma, 1997). 35S::AG ful apl cal plants (D) did not flower even after several months of growth. The inflorescences resemble those of ful ap1 cal triple mutants, but the leafy organs are reduced in size and curled upwards, a typical effect of the ectopic $A G$ activity.

by its complex pattern of expression (Mandel and Yanofsky, 1995a). FUL has an early function in controlling flowering time, meristem identity and cauline leaf morphology, and has a later role in carpel and fruit development that affects valve, replum and style morphology. These phenotypic effects correlate well with the biphasic pattern of FUL expression $(\mathrm{Gu}$ et al., 1998; Mandel and Yanofsky, 1995a) and reveal a certain degree of non-autonomy of the FUL function since the mutants have a clear phenotype in the replum region where no FUL RNA is detected. Moreover, the study of an allelic series shows that these different roles are separable. All of the mutant alleles have a similar effect on flowering time (C. F. and M. Y., unpublished observations) and cauline leaf morphology. However, the weak alleles display a much less severe phenotype in valve and replum morphology as compared to strong alleles.

\section{$A P 1 / C A L / F U L$ act redundantly to upregulate $L F Y$ in floral meristems}

It has been proposed that a threshold level of $L F Y$ expression is required for the vegetative-to-floral transition (Blázquez et al., 1997). Our results indicate that this threshold level is not reached in ful apl cal plants, leading to a dramatic nonflowering phenotype. The initial activation of $L F Y$ does not depend on $F U L / A P 1 / C A L$, but these activities are necessary for its subsequent upregulation, since $L F Y$ expression in ful apl cal meristems never exceeds its initially low levels. Moreover, constitutive expression of $L F Y$ restored flowering in the ful apl cal plants, reinforcing the idea of $L F Y$ levels as the switch to reproductive development. Our results agree with previous studies that identified $A P I$ and $C A L$ as direct or indirect activators of $L F Y$. These studies showed that $L F Y$ is ectopically expressed in the converted flower meristems of 35S::AP1 plants (Liljegren et al., 1999) and that the initial expression of $L F Y$ is significantly reduced in apl cal mutants (Bowman et al., 1993).

It is interesting to note that although constitutive expression of $L F Y$ suppressed the non-flowering phenotype of ful apl cal triple mutants, many of the phenotypes caused by the ectopic $L F Y$ expression were suppressed in the triple mutant background. It has been previously reported that the flower-toshoot conversion in 35S::LFY plants is largely suppressed by mutations in $A P 1$, although the plants are still early flowering (Weigel and Nilsson, 1995). We found that the flowering time in 35S::LFY apl cal plants is further increased and that 35S::LFY ful apl cal plants flower significantly later than wild-type plants (Table 2). This suggests that the threshold of $L F Y$ required to induce flowering is higher in the ful apl cal background, or alternatively, that another factor may be required to accumulate when $A P 1 / C A L / F U L$ are not present, to act with $L F Y$ in promoting flowering.

Similar phenotypes to those found in ful apl cal plants have been reported for several mutant combinations. For example, the ft apl and fwa apl double mutant inflorescences resemble the ful apl cal 'leafy cauliflowers', although they are able to flower after several months of growth (Ruiz-García et al., 1997). Similarly, ld apl cal triple mutants form proliferating leafy shoots at the apex and are unable to flower (Aukerman et al., 1999). The apl cal double mutants also show an enhancement of vegetative characteristics when grown in short days or at $16^{\circ} \mathrm{C}$ (Bowman et al., 1993). $L F Y$ fails to be upregulated both in ld apl cal and apl cal grown in noninductive conditions (Aukerman et al., 1999; Bowman et al., 1993). The similarities among these mutant phenotypes and the ful apl cal inflorescences may reflect a possible role of the FT, $F W A$ and $L D$ genes in the competence of the meristems to respond to reduced levels of $L F Y$, and/or indicate their possible function as $L F Y$ activators. It will be interesting to test whether $F U L$ fails to be upregulated at low temperatures or in the $f t$, $f w a$ or $l d$ backgrounds, thereby preventing $L F Y$ activation.

\section{AP1/CAL/FUL may control the transition between developmental phases by modulating the ratio of LFY/TFL activities}

It has been suggested that the overlapping expression domains of $L F Y$ and TFLI in emerging lateral primordia cause the meristem proliferation of apl cal inflorescences (Ratcliffe et al., 1999). Support for this idea comes from genetic studies, 
which show that this proliferation does not occur when lfy or tfll mutations are introduced into the apl cal double mutant. Thus, the role for $C A L$ in an apl mutant background is to activate $L F Y$ and repress $T F L 1$ expression in lateral meristems, thus preventing their overlapping activities (Bowman et al., 1993; Ratcliffe et al., 1999).

We have found that the expression domains of $L F Y$ and TFL1 overlap in the ful apl cal triple mutants, as was observed for apl cal meristems (Ratcliffe et al., 1999; this work). Accordingly, the ful apl cal plants formed meristems in a reiterate pattern, and this proliferation was not observed when $L F Y$ or $T F L$ were also mutated. In the apl cal background, where $F U L$ is expressed at the apex and ectopically in all lateral meristems, $F U L$ was required for the increase of $L F Y$ expression, whereas it seemed to have little effect on TFL1 regulation. Thus, in the ful apl cal inflorescences, the ratio of LFY:TFL1 expression was always lower than in apl cal double mutants.

Our data suggest that the levels of $L F Y$ as well as the relative levels of $L F Y$ and TFLI control plant architecture and meristem behavior. When apl cal SAMs enter the inflorescence developmental phase, they give rise laterally to new meristems that also behave as inflorescence apical meristems. In ful ap1 cal plants, the SAMs seem to proliferate as $\mathrm{V}_{2}$ meristems, producing cauline leaves with axillary meristems that in turn repeat this pattern forming the 'leafy cauliflowers' (Figs 2, 3). Thus, lower $L F Y$ to $T F L 1$ relative levels, together with their overlapping expression, would result in the reiteration of a more vegetative phase in ful apl cal plants. In contrast, in apl cal double mutants, a slightly higher $L F Y$ to $T F L 1$ ratio, together with higher $L F Y$ levels, would allow the transition to the inflorescence phase, that in turn would cause the reiterative behavior of the meristems. The subsequent upregulation of $L F Y$ in apl cal, mediated by $F U L$, would raise the $L F Y$ :TFL1 ratio to the required levels for floral specification. The late formation of floral structures in ful apl cal inflorescences in a TFL1 heterozygous background seems to support this hypothesis, since in this situation, a reduced TFL1 activity could eventually lead to the transition to floral commitment.

As noted above, the proliferating ful apl cal meristems appear to be arrested in the $\mathrm{V}_{2}$ phase, in contrast to the reproductive character of the apl cal cauliflowers. Besides the morphological evidence, additional data support this conclusion. For example, constitutive $A G$ expression failed to promote floral identity in the $f u l$ apl cal meristems whereas it was able to promote flowering in apl cal double mutants (Mizukami and Ma, 1997). The lack of floral specification in 35S::AG ful apl cal plants suggests that $A G$ may act through $A P 1 / C A L / F U L$ to promote floral fate, or, alternatively, that the ful apl cal background prevents the meristems from becoming competent to respond to $A G$, perhaps by keeping them in a vegetative state.

\section{FUL has a floral promoting activity independent of LFY}

We have demonstrated that $F U L$ plays a redundant role with $A P 1$ and $C A L$ in $L F Y$ upregulation, thus promoting floral meristem specification. Our data also suggest that $F U L$ is involved in phase transition during development in a pathway that is independent of $L F Y$. This is clearly illustrated by the observation that the delay in flowering caused by mutations in $L F Y$ is further enhanced in ful lfy double mutants (Table 1). In addition, small but significant increases in $\mathrm{V}_{1}$ and $\mathrm{V}_{2}$ phases are found in ful single mutants, even though levels of $L F Y$ expression are not noticeably affected. Furthermore, flowering time is dramatically reduced in plants that constitutively
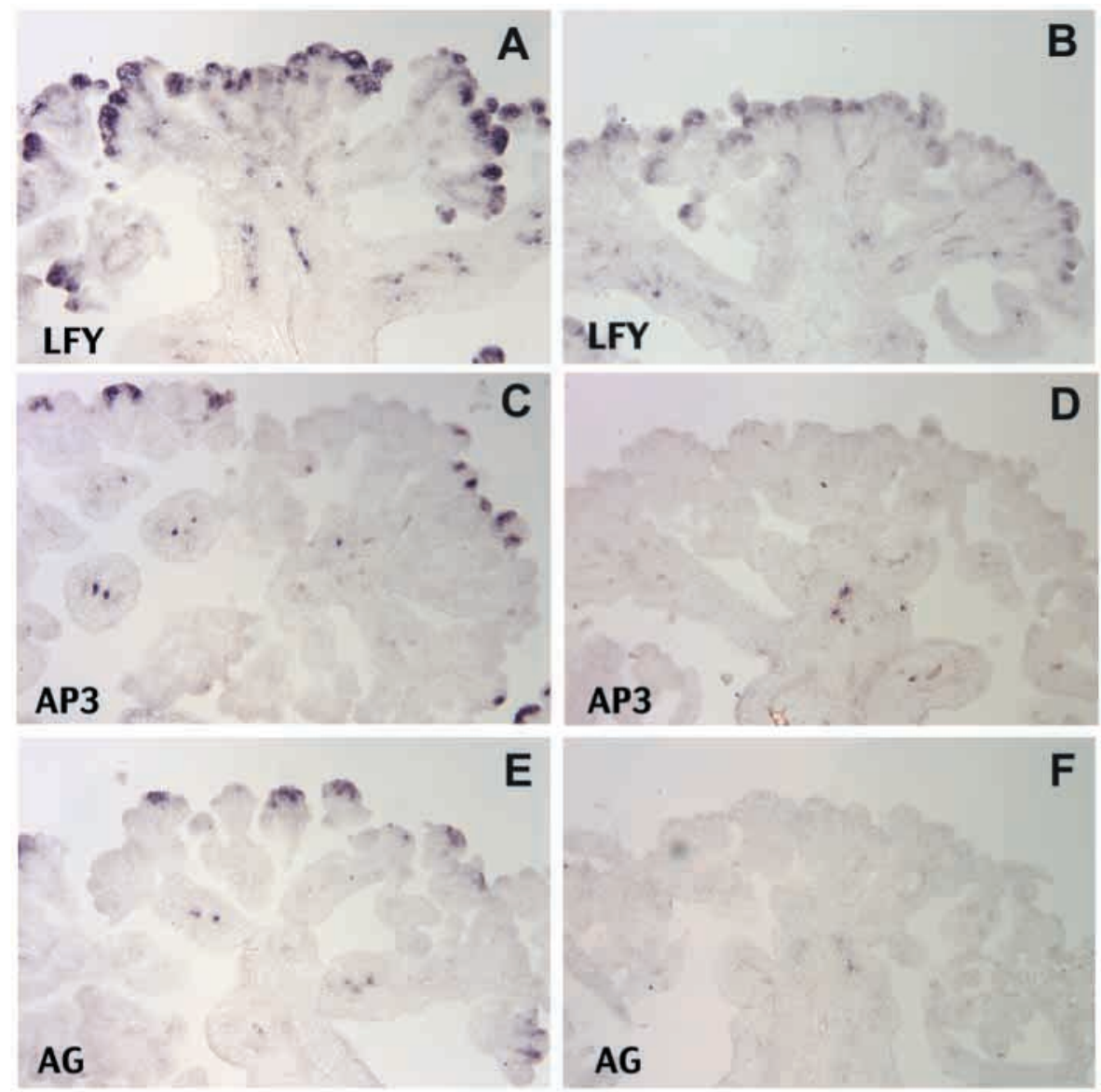

ap1 cal

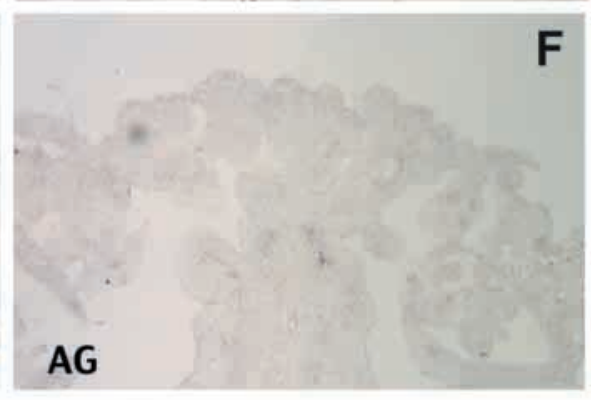

ful ap1 cal

Fig. 6. Expression of $L F Y, A P 3$ and $A G$ in apl cal and ful apl cal plants. Sections of 21-dayold apl cal (A,C,E) and ful apl cal (B,D,F) plants probed with $L F Y(\mathrm{~A}, \mathrm{~B}), A P 3(\mathrm{C}, \mathrm{D})$ and $A G(\mathrm{E}, \mathrm{F})$ antisense RNA are shown. Sections in $\mathrm{A}, \mathrm{C}, \mathrm{E}$ and $\mathrm{B}, \mathrm{D}, \mathrm{F}$ are from single inflorescences. $L F Y$ is strongly expressed in the presumed floral meristems of apl cal inflorescences. In ful apl cal meristems, $L F Y$ is detected at much reduced levels. $(\mathrm{C}, \mathrm{G})$ Some meristems in apl cal inflorescences show $A P 3(\mathrm{C})$ and $A G(\mathrm{E})$ patterns of expression similar to those found in early stages (4-5) of wild-type development. (D,F) AP3 (D) and $A G(\mathrm{~F})$ are not detected in ful apl cal meristems. 
express FUL under the control of the $35 \mathrm{~S}$ promoter, and this floral-promoting activity is independent of $L F Y$, since is not affected in the lfy mutant background (C.F. and M.F.Y., unpublished results). It is interesting to note that the $L F Y$ independent role of $F U L$ in promoting the phase transition requires $A P 1$, since $F U L$ is unable to promote flowering in a lfy apl background. However the small effect on flowering time caused by ful mutations, and the rapid and strong $F U L$ upregulation in the SAM after the induction of the reproductive phase, suggest that its flower promoting activity might be largely obscured by other highly redundant activities. Good candidates for genes that act redundantly with FUL in the SAM are the AGL2O and AGL24 MADS-box genes that share a similar pattern of upregulation (S. Gold and M. Yanofsky; C. Gustafson-Brown, M. Yanofsky and W. Crosby; unpublished results). Regardless of whether additional MADS-box genes or as yet unidentified genes are involved, it is clear that FUL acts in a highly redundant pathway to control the transition to flowering.

We thank Soraya Pelaz and Miguel A. Blázquez for helpful discussions and, together with Allen Sessions, Medard $\mathrm{Ng}$ and Detlef Weigel, for critical reading of the manuscript. Detlef Weigel, Hong Ma, Judy Roe, Allen Sessions, Ellen Wisman, David Smyth, John Bowman and Sarah Liljegren for providing seeds, Pilar Cubas for information on double labeling in situ experiments, and Tony Gaba, Amy Chen, Cheryl Wiley and Tad Kawashiwa for excellent technical assistance. This work was supported by grants from the National Science Foundation and the National Institutes of Health to M. F. Y. C. F. was a recipient of a postdoctoral fellowship from the Spanish Ministry of Education and Science.

\section{REFERENCES}

Alvarez, J., Guli, C. L., Yu, X.-H. and Smyth, D. R. (1992). terminal flower, a gene affecting inflorescence development in Arabidopsis thaliana. Plant J. 2, 103-116.

Aukerman, M. J., Lee, I., Weigel, D. and Amasino, R. (1999). The Arabidopsis flowering-time gene LUMINIDEPENDENS is expressed primarily in regions of cell proliferation and encodes a nuclear protein that regulates $L E A F Y$ expression. Plant J. 18, 195-203.

Blázquez, M. A., Soowal, L., Lee, I. and Weigel, D. (1997). LEAFY expression and flower initiation in Arabidopsis. Development 124, 38353844.

Bowman, J. L., Alvarez, J., Weigel, D., Meyerowitz, E. M. and Smyth, D. R. (1993). Control of flower development in Arabidopsis thaliana by APETALA1 and interacting genes. Development 119, 721-743.

Bradley, D. J., Ratcliffe, O. J., Vincent, C., Carpenter, R. and Coen, E. S. (1997). Inflorescence commitment and architecture in Arabidopsis. Science 275, 80-83.

Drews, G. N., Bowman, J. L. and Meyerowitz, E. M. (1991). Negative regulation of the Arabidopsis homeotic gene AGAMOUS by the APETALA2 product. Cell 65, 991-1002.

Gu, Q., Ferrándiz, C., Yanofsky, M. F. and Martienssen, R. (1998). The FRUITFULL MADS-box gene mediates cell differentiation during Arabidopsis fruit development. Development 125, 1509-1517.

Hempel, F. D., Weigel, D., Mandel, M. A., Ditta, G., Zambryski, P.,
Feldman, L. J. and Yanofsky, M. F. (1997). Floral determination and expression of floral regulatory genes in Arabidopsis. Development 124, 3845-3853.

Huala, E. and Sussex, I. M. (1992). LEAFY interacts with floral homeotic genes to regulate Arabidopsis floral development. Plant Cell 4, 901-913.

Irish, V. F. and Sussex, I. M. (1990). Function of the apetala-1 gene during Arabidopsis floral development. Plant Cell 2, 741-751.

Jack, T., Brockman, L. L. and Meyerowitz, E. M. (1992). The homeotic gene APETALA3 of Arabidopsis thaliana encodes a MADS-box and is expressed in petals and stamens. Cell 68, 683-697.

Kempin, S. A., Savidge, B. and Yanofsky, M. F. (1995). Molecular basis of the cauliflower phenotype in Arabidopsis. Science 267, 522-525.

Lee, I., Wolfe, D. S., Nilsson, O. and Weigel, D. (1997). A $L E A F Y$ coregulator encoded by UNUSUAL FLORAL ORGANS. Curr. Biol. 7, 95-104.

Liljegren, S., Gustafson-Brown, C., Pinyopich, A., Ditta, G. and Yanofsky, M. (1999). Interactions among the meristem identity genes APETALAl, LEAFY and TERMINAL FLOWER specify meristem fate. Plant Cell 11, 1007-1018.

Mandel, M. A., Gustafson-Brown, C., Savidge, B. and Yanofsky, M. F. (1992). Molecular characterization of the Arabidopsis floral homeotic gene APETALA1. Nature 360, 273-277.

Mandel, M. A. and Yanofsky, M. F. (1995a). The Arabidopsis AGL8 MADS box gene is expressed in inflorescence meristems and is negatively regulated by APETALA1. Plant Cell 7, 1763-1771.

Mandel, M. A. and Yanofsky, M. F. (1995b). A gene triggering flower development in Arabidopsis. Nature 377, 522-524.

Mizukami, Y. and Ma, H. (1992). Ectopic expression of the floral homeotic gene AGAMOUS in transgenic Arabidopsis plants alters floral organ identity. Cell 71, 119-131.

Mizukami, Y. and Ma, H. (1997). Determination of Arabidopsis floral meristem identity by AGAMOUS. Plant Cell 9, 393-408.

Neff, M., Neff, J., Chory, J. and Pepper, A. (1998). dCAPS, a simple technique for the genetic analysis of single nucleotide polymorphisms:experimental aplications in Arabidopsis thaliana genetics. Plant J. 14, 387-392.

Piñeiro, M. and Coupland, G. (1998). The control of flowering time and floral identity in Arabidopsis. Plant Physiol. 117, 1-8.

Poethig, R. S. (1990). Phase changes and the regulation of shoot morphogenesis in plants. Science 250, 923-930.

Ratcliffe, O., Bradley, D. and Coen, E. (1999). Separation of shoot and floral identity in Arabidopsis. Development 126, 1109-1120.

Ruiz-García, L., Madueño, F., Wilkinson, M., Haughn, G., Salinas, J. and Martínez-Zapater, J. M. (1997). Different roles of flowering time genes in the activation of floral initiation genes in Arabidopsis. Plant Cell 9, 19211934.

Savidge, B. (1996). Floral meristem specification and floral organ development in Arabidopsis. University of California at San Diego, La Jolla, CA.

Schultz, E. A. and Haughn, G. W. (1991). LEAFY, a homeotic gene that regulates inflorescence development in Arabidopsis. Plant Cell 3, 771-781.

Shannon, S. and Meeks-Wagner, D. R. (1991). A mutation in the Arabidopsis TFL1 gene affects inflorescence meristem development. Plant Cell 3, 877892 .

Shannon, S. and Meeks-Wagner, D. R. (1993). Genetic interactions that regulate inflorescence development in Arabidopsis. Plant Cell 5, 639-655.

Smyth, D. R., Bowman, J. L. and Meyerowitz, E. M. (1990). Early flower development in Arabidopsis. Plant Cell 2, 755-767.

Weigel, D., Alvarez, J., Smyth, D. R., Yanofsky, M. F. and Meyerowitz, E. M. (1992). $L E A F Y$ controls floral meristem identity in Arabidopsis. Cell 69, 843-859.

Weigel, D. and Nilsson, O. (1995). A developmental switch sufficient for flower initiation in diverse plants. Nature 377, 495-500.

Yanofsky, M. (1995). Floral meristems to floral organs: genes controlling early events in Arabidopsis flower development. Annu. Rev. Plant Physiol. Plant Mol. Biol., 46, 167-188. 